\title{
Women with Polycystic Ovarian Syndrome Exhibit Reduced Baroreflex Sensitivity That May Be Associated with Increased Body Fat
}

\author{
Stella Vieira Philbois, ${ }^{\circledR}$ Ada Clarice Gastaldi, ${ }^{\circledR}$ Tábata de Paula Facioli, ${ }^{\circledR}$ Ana Carolina Sanches Felix, ${ }^{\circledR}$ \\ Rosana Maria dos Reis, ${ }^{(10}$ Thauane Hanna Fares, Hugo Celso Dutra de Souzaํㅜㄹ \\ Faculdade de Medicina de Ribeirão Preto, Ribeirão Preto, SP - Brazil
}

\begin{abstract}
Background: Polycystic ovarian syndrome (PCOS) women have a high prevalence of obesity and alterations in cardiovascular autonomic control, mainly modifications in heart rate variability (HRV) autonomic modulation. However, there are few studies about other autonomic control parameters, such as blood pressure variability (BPV) and baroreflex sensitivity (BRS). In addition, there are still doubts about the obesity real contribution in altering autonomic control in these women.

Objective: To investigate BPV and BRS autonomic modulation alterations in PCOS women, as well as, to evaluate whether these alterations are due PCOS or increased body fat.

Methods: We studied 30 eutrophic volunteers [body mass index (BMI) $<25 \mathrm{~kg} / \mathrm{m}^{2}$ ] without PCOS (control group) and 60 volunteers with PCOS divided into: eutrophic (BMI $\left.<25 \mathrm{~kg} / \mathrm{m}^{2}, \mathbf{N}=30\right)$ and obese women $\left(\mathrm{BMI}>30 \mathrm{~kg} / \mathrm{m}^{2}, \mathrm{~N}=30\right)$. All volunteers were submitted to anthropometric evaluation, hemodynamic and cardiorespiratory parameters record at rest and during physical exercise, analysis of HRV, BPV and spontaneous BRS. The differences in p less than $5 \%$ (p < 0.05 ) were considered statistically significant.

Results: Related to eutrophics groups, there were no differences in autonomic parameters evaluated. The comparison between the PCOS groups showed that both PCOS groups did not differ in the BPV analysis. Although, the obese PCOS group presented lower values of spontaneous BRS and HRV, in low frequency and high frequency oscillations in absolute units.
\end{abstract}

Conclusion: Our results suggest that obesity did little to alter HRV in women with PCOS, but it may influence the spontaneous BRS. (Arq Bras Cardiol. 2019; 112(4):424-429)

Keywords: Obesity; Hypertension; Polycystic Ovary Syndrome/physiopathology; Adiposity; Body Fat Distribution; Autonomic Nervous System; Heart Rate.

\section{Introduction}

Women with polycystic ovarian syndrome (PCOS) frequently present cardiovascular autonomic control impairments, mainly characterized by a cardiac autonomic imbalance in determining heart rate variability (HRV). ${ }^{1-4}$ This imbalance is an important cardiovascular diseases risk predictor. ${ }^{5-7}$ The autonomic impairment causes are still not well established. Some studies suggest that they are result of hormonal and metabolic disorders due PCOS, such as insulin resistance increased..$^{2,3,8}$ On the other hand, it is possible that they are simply due body fat percentage increase, which triggers series of systemic alterations, including metabolic and cardiovascular, that affect the cardiac autonomic control. , $, 9,10$

Mailing Address: Hugo Celso Dutra de Souza •

Rua Luís Basso, 130. Postal Code 14040-150, Jardim Recreio, Ribeirão Preto, SP - Brazil

E-mail: hugocds@fmrp.usp.br, hugousp@gmail.com

Manuscript received May 08, 2018, revised manuscript July 25, 2018, accepted August 15, 2018

DOI: $10.5935 / a b c .20190031$
Another important aspect is that only HRV is frequently investigated in these women, and we know little about PCOS effects on others autonomic parameters, such as baroreflex sensitivity (BRS) and blood pressure variability (BPV). More specifically, there are no studies associating PCOS to BPV, and in BRS case, studies are incipient. On this, only one study was performed and found no differences. ${ }^{11}$ However, this study only addressed obese PCOS and non-PCOS women, which limited further findings.

Therefore, the aim of the present study was to evaluate spontaneous BRS and BPV in eutrophic PCOS women and to investigate the contribution of obesity to these autonomic parameters in these women.

\section{Methods}

\section{Participants}

With a convenience sample, ninety volunteers aged between 18 and 39 years were included, 30 non PCOS women, considered as a control group, and 60 PCOS women, according to Rotterdam consensus, ${ }^{12}$ were subdivided according to the body mass index (BMI): eutrophic group (30 women) and obese group (30 women). All of them were sedentary, did not 
use any medication, and were screened at the outpatient clinic of the Gynecology and Obstetrics Clinic of Clinical Hospital of Ribeirao Preto Medical School (HC-FMRP/USP).

\section{Polycystic ovary syndrome diagnostic}

Transvaginal pelvic ultrasound was performed with the Voluson 730 Expert Machine (GE Medical Systems, ZIPF, Austria) to analysis the cysts presence or absence. The ovarian volume and follicles number/size were evaluated, and to calculate ovarian volume the prolate ellipsoid formula (depth $x$ width $x$ length $x$ 0.5) was used. ${ }^{13}$

In addition, laboratory tests for serum total testosterone, androstenedione, sex hormone binding globulin and free androgen, prolactin, 17-hydroxyprogesterone and thyrotropin dosed to diagnose exclusion causes. Blood samples were collected during the follicular phase in women with regular ovulatory cycles and at any time in those with irregular cycles. All the above examinations were performed at the Gynecology Laboratory of HC-FMRP, between 07h00 and 09h00 a.m. after a previous 12-hour fast.

\section{Ergospirometric test}

The peak oxygen uptake $\left(\mathrm{VO}_{2 \text { peak }}\right)$ was assessed by a submaximal exercise test on a treadmill (Super ATL Millenium ${ }^{\circledR}$, Inbramed/Inbrasport, Brazil) using the Modificated Bruce protocol. The analysis of exhaled gases $\left(\mathrm{VO}_{2}\right.$ and $\left.\mathrm{VCO}_{2}\right)$ was performed using a metabolic device (UltimaTM CardiO², Medical Graphics Corp., USA).

\section{Anthropometric parameters}

Body weight and height were obtained using an analogue scale with an altimeter (Welmy), while the body mass index (BMI) values were obtained using the formula $\mathrm{W} / \mathrm{H}^{2}$, where $\mathrm{W}$ is the weight in kilograms and $\mathrm{H}$ is the height of the subject in meters. Body composition was evaluated using the bioelectrical impedance method (Quantum BIA 101; Q-RJL Systems, Clinton Township, Michigan, USA). The groups were subdivided by their $\mathrm{BMI}$, where the eutrophic groups had $\mathrm{BMI}<25 \mathrm{~kg} / \mathrm{m}^{2}$ and the obese group had BMI $>30 \mathrm{~kg} / \mathrm{m}^{2} \cdot{ }^{14}$

Analysis of the heart rate variability and blood pressure variability

The spectral analysis of HRV was recorded between 09h00 and 10 h00 a.m. according to the following protocol: after remaining in a supine rest position on orthostatic bed for $20 \mathrm{~min}$, the volunteers were passively placed in an inclined position $\left(75^{\circ}\right.$ angle) for an additional $10 \mathrm{~min}$. HRV for supine and inclined positions (that is, the tilt test) was recorded using an electrocardiogram (AD Instruments, Sydney, Australia), and a time series of RR interval (RRi) was obtained.

The HRV was obtained using the RRi from electrocardiographic record (ECG), through the modified MC5 shunt at a sampling frequency of $1000 \mathrm{~Hz}$. The BPV data values were obtained from the systolic arterial pressure (SAP) recorded beat-to-beat by means of digital plethysmography recording equipment, FINOMETER (Finometer Pro, Finapress Medical System,
Amsterdam, Netherland). The room temperature was kept at $21^{\circ} \mathrm{C}$, the ambient light and the noise were controlled, to prevent any interference with recording of data.

The BPV and HRV analyses were performed using custom computer software (CardioSeries v2.0, http://sites.google.com/ site/cardioseries). The values of the RRi and SAP intervals were redesigned in $3 \mathrm{~Hz}$ cubic spline interpolation, to normalize the time interval between the beats. The series of interpolated RRi and SAP follow the Welch Protocol; $;^{15}$ they have been divided into half-overlapping sets of 256 data points, overlapping $50 \%$. The stationary segment was visually inspected and those with artifacts or transients were excluded. Each RRi and SAP stationary segment were submitted to spectral analysis by Fast Fourier Transform (FFT), after Hanning window. The RRi specters were integrated in low frequency (LF; $0.04-0.15 \mathrm{~Hz}$ ) and high frequency (HF; $0.15-0.5 \mathrm{~Hz}$ ) bands and the results are expressed in absolute $\left(\mathrm{ms}^{2}\right)$ and normalized units (nu), while the SAP specters were integrated only in low frequency band $(\mathrm{LF} ; 0,04-0,15 \mathrm{~Hz})$ and the results are expressed in absolute units $\left(\mathrm{mmHg}^{2}\right)$.

The HRV normalized values were obtained by calculating the percentage of LF and HF power related to the total power of spectrum minus the very low-frequency band $(\mathrm{VLF} ;<0.2 \mathrm{~Hz}){ }^{16,17}$ In addition, normalization procedure was performed to minimize variations of total power in the absolute value of LF and HF. ${ }^{18}$ To assess the sympathovagal balance, LF/HF ratio of RRi variability was also calculated. ${ }^{19}$

\section{Spontaneous baroreflex sensitivity}

The BRS was assessed in time-domain using the sequence technique, as described by Di Rienzo et al., ${ }^{20}$ The computer software CardioSeries v2.4 scanned beat-to-beat time series of RRi and SAP values searching for sequences of at least 3 consecutive beats in which; progressive increases in SAP were followed by progressive increases in RRi (up sequence) and progressive decreases in SAP were followed by progressive decreases in RRi (down sequence), with a correlation coefficient ( $r$ ) between RRi and SAP values higher than 0.8. The mean slope of the linear regression line between the SAP and RRi values of each sequence found determined spontaneous BRS.

\section{Statistical analysis}

In a comparison between two groups the Student's t-test and in comparison of three groups the one ways variance analysis (ANOVA ONE WAY) were performed. The Shapiro-Wilk test was used to verify de the dates normality; when the distribution was not normal, non-parametric tests were used, the Mann-Whitney test to compare between two groups, and in comparison of three groups, the Kruskal-Wallis test. When the variables had a normal distribution, they were described as mean ( \pm standard deviation), and which had non-parametric distribution they were described as median ( \pm interquartile range). The differences in $p$ were less than $5 \%$ $(p<0.05)$ were considered statistically significant. All statistical tests were performed with Sigma Stat 3.5 software (Systat Software Inc., San Jose, CA, USA). 


\section{Results}

The volunteer's anthropometric characteristics and hemodynamic parameters are in Table 1. The obese PCOS group had higher $\mathrm{BMI}$, weight and body fat percentage than the other groups. On the other hand, $\mathrm{VO}_{2 \text { peak }}$ was lower in the obese PCOS group. In relation to blood pressure, the obese group had higher values of diastolic blood pressure and mean blood pressure compared to the control and eutrophic PCOS groups.

Table 2 presents the spectral analysis of HRV and BPV results during rest of all groups studied. The HRV analysis at rest shows the obese PCOS group had lower variance. In addition, the control groups and eutrophic PCOS presented higher LF and HF oscillations in absolute values than the obese PCOS group. There were no differences between the groups in BPV analysis.

The results of BRS analysis obtained during rest in all groups studied, control, eutrophic PCOS and obese PCOS, are seen in Table 3, that show at rest the obese PCOS group presented lower spontaneous BRS than the others groups. In addition, it is important to note that the control group demonstrated a higher baroreflex effectiveness index.

\section{Discussion}

The present study mainly findings were, at rest the obese PCOS group had lower HRV and BRS than the other two groups, BPV was similar across groups.

Regarding hemodynamic values, PCOS obese group showed the highest values of systolic, diastolic and mean blood pressure compared to other groups, despite the fact that all subjects were normotensive; some studies had also show a relation with body fat increase and increase BP values. . $^{90,21,22}$ To $\mathrm{VO}_{2 \text { peak }}$ the obese PCOS group had the lowest value, similarity to literature, which some authors found a negative correlation between obesity and VO2peak. ${ }^{21,22}$

There are few studies in the literature about obesity and PCOS, which are contradictory, some point to this association as a negative factor in $\mathrm{HRV}^{3,4}$ although others report that there is no association between weight gain and PCOS. ${ }^{11,23}$ In this sense, the lower HRV found in the obese PCOS group in the present study suggests that this change is due to obesity. The literature indicates that the obesity mechanisms may be associated with a reduced sympathetic system response in the postsynaptic region since they had found in presynaptic cleft a high sympathetic activity represented by high concentration of noradrenaline. ${ }^{24,25}$ In addition, a recent study carried out in our laboratory showed low frequency (LF) and high frequency (HF) bands differences, in absolute and normalized units, in healthy and sedentary women with normal BMI, overweight and obesity, they verified that the obese group had lower LF and HF oscillations. ${ }^{10}$

Regarding BRS, the eutrophic PCOS and control groups presented similar values, agreeing with Lambert, 2015, in which the groups had similar BMI and BRS values. In relation to the obese PCOS group, it had the lowest values in all BRS parameters than the others two eutrophic groups, suggesting that obesity may be responsible for a reduction in BRS. In this sense, a study comparing BRS in women divided by $\mathrm{BMI}$ indicates a BRS reduction with gain weight, observed by the BRS gain value, in this way, the BRS decrease might correlate to weight increase. ${ }^{26}$ However, it is known that BRS is also influenced by many other factors like insulin resistance, blood glucose, sodium sensibility, genetic markers and ovarian hormones. ${ }^{27,28}$ In the present study, neither of these other factors were measuring. Thereby it is possible to suggest that obesity may influenced in BRS values, as observed in another study, ${ }^{26}$ although further studies are needed to confirm these findings in PCOS women.

Table 1 - Hemodynamic characteristics and values among healthy women and women with polycystic ovary syndrome (PCOS), subdivided into eutrophic PCOS $\left(\mathrm{BMI}<25 \mathrm{~kg} / \mathrm{m}^{2}\right)$ and obese PCOS $\left(\mathrm{BMI}>30 \mathrm{~kg} / \mathrm{m}^{2}\right)$

\begin{tabular}{|c|c|c|c|c|c|}
\hline & Control & PCOS eutrophic & PCOS obese & $\mathbf{p}^{\prime}$ & $p^{\prime \prime}$ \\
\hline \multicolumn{6}{|l|}{ Characteristics } \\
\hline Age, years & $31.2 \pm 6.6$ & $28.5 \pm 5.2$ & $30.2 \pm 5.3$ & 0.053 & 0.107 \\
\hline Heights, meters & $1.64 \pm 5.0$ & $1.62 \pm 5.8$ & $1.62 \pm 7.9$ & 0.102 & 0.649 \\
\hline Weight, kg & $64 \pm 10$ & $60.6 \pm 5.7$ & $90.3 \pm 10.9^{*}+$ & 0.09 & $<0.001$ \\
\hline BMl, kg/m² & $23.5 \pm 3$ & $22.9 \pm 1.6$ & $33.9 \pm 2.4^{*}+$ & 0.494 & $<0.001$ \\
\hline Body fat percentage, $\%$ & $25.6 \pm 3.6$ & $26.4 \pm 3.4$ & $44.3 \pm 3.3^{* \dagger}$ & 0.325 & $<0.001$ \\
\hline $\mathrm{VO}_{2 \text { peak }}, \mathrm{L} / \mathrm{min} / \mathrm{kg}$ & $35.5 \pm 3.3$ & $31.9 \pm 3.9$ & $25.3 \pm 3.3^{* \dagger}$ & 0.05 & $<0.001$ \\
\hline \multicolumn{6}{|l|}{ Hemodynamics Values } \\
\hline $\mathrm{HR}$ (bpm) & $76 \pm 2.6$ & $74.6 \pm 2$ & $77 \pm 2$ & 0.764 & 0.416 \\
\hline $\mathrm{SBP}(\mathrm{mmHg})$ & $105 \pm 8.9$ & $101 \pm 11.8$ & $111 \pm 9.5^{\dagger}$ & 0.057 & $<0.001$ \\
\hline $\mathrm{DBP}(\mathrm{mmHg})$ & $70 \pm 10.3$ & $66 \pm 9.6$ & $76 \pm 7.4^{* \dagger}$ & 0.05 & $<0.001$ \\
\hline $\operatorname{MBP}(\mathrm{mmHg})$ & $84 \pm 9$ & $80 \pm 9.8$ & $90 \pm 7.5^{5 \dagger}$ & 0.05 & $<0.001$ \\
\hline
\end{tabular}

Values expressed as means $\pm S D$ : standard deviation; $m$ : Meters; $\mathrm{Kg}$ : kilogram; BMI: body mass index; $\mathrm{VO}_{2 \text { poak }}$ : volume of oxygen consumed at the peak of exercise; L/min/Kg: liters per minutes per kilo; HR: heart rate; bpm: beat per minute; SBP: systolic blood pressure; DBP: diastolic blood pressure; MBP: mean blood pressure; mmHg: millimeters of mercury; statistical difference when $p<0.05 ;\left(^{*}\right)$ vs. Control; $(\dagger)$ vs. eutrophic PCOS; $P^{\prime}$ : eutrophic control group vs PCOS eutrophic group; $P^{\prime \prime}$ : PCOS eutrophic group vs. PCOS obese group. 
Table 2 - Parameters of the spectral analysis of the heart rate variability analysis calculated from the time series RR intervals and systolic arterial pressure variability calculated by the pulse beat-to-heart rate interval obtained between women without and with polycystic ovaries syndrome (PCOS) divided according to the body mass index eutrophic $<25 \mathrm{~kg} / \mathrm{m}^{2}$ and obese $>30 \mathrm{~kg} / \mathrm{m}^{2}$

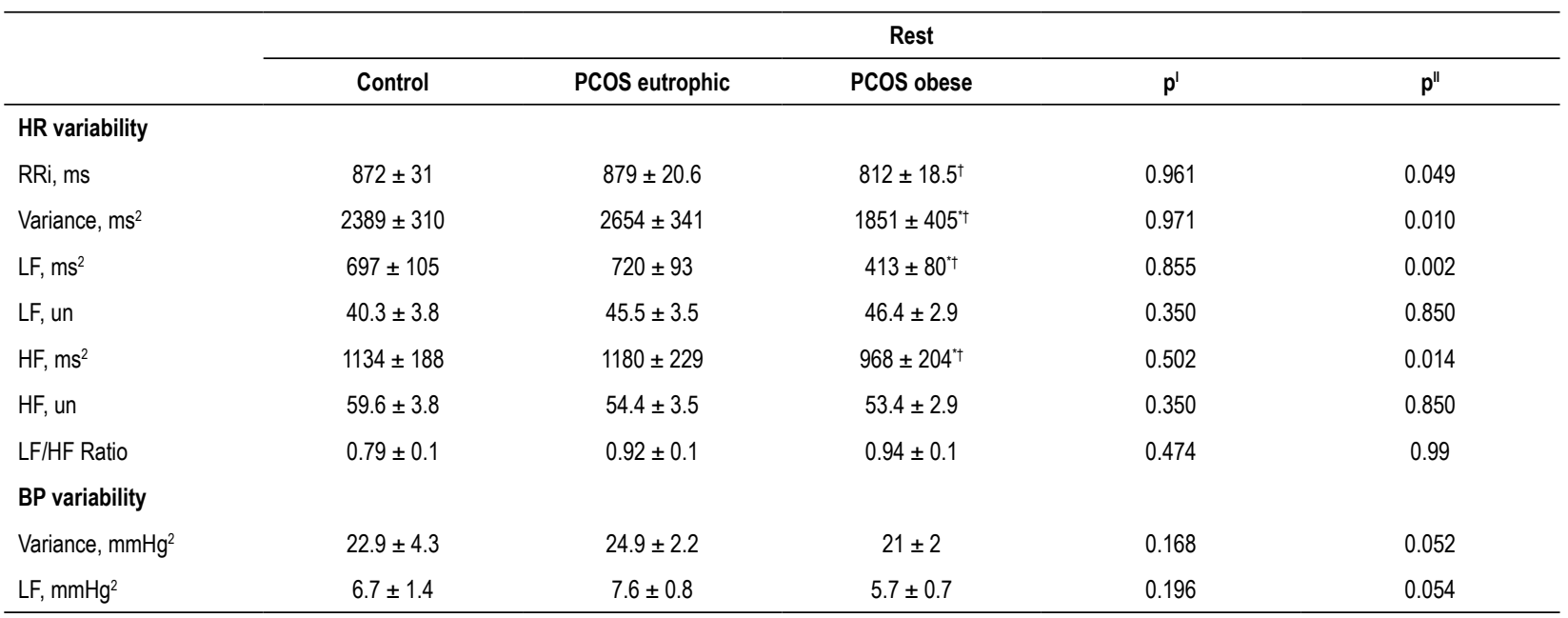

Values expressed as means $\pm S D$ : standard deviation; HR: heart rate; RRi: interval between $R$ waves on the electrocardiogram; nu: normalized units; $m s^{2}:$ milliseconds squared; LF: low frequency band; HF: high frequency band; BP: blood pressure; significant difference $p<0.05$; ( ${ }^{*}$ ) vs rest control, (t) vs. rest eutrophic PCOS; $P^{l}$ : eutrophic control group vs PCOS eutrophic group; Pll: PCOS eutrophic group vs. PCOS obese group.

Table 3 - Parameters of the baroreflex analysis by the calculated sequence series of RR intervals obtained between women with and without polycystic ovary syndrome (PCOS) divided according to the body mass index eutrophic $<25 \mathrm{~kg} / \mathrm{m}^{2}$ and obese $>30 \mathrm{~kg} / \mathrm{m}^{2}$

\begin{tabular}{|c|c|c|c|c|c|}
\hline & \multicolumn{5}{|c|}{ Rest } \\
\hline & Control & PCOS eutrophic & PCOS obese & $p^{\prime}$ & $p^{\prime \prime}$ \\
\hline \multicolumn{6}{|c|}{ Baroreflex Sensitivity } \\
\hline Ramp numbers & $85 \pm 40.7$ & $84.3 \pm 39.8$ & $93.7 \pm 42.4$ & 0.853 & 0.379 \\
\hline BEI & $0.74 \pm 0.13$ & $0.63 \pm 0.12^{*}$ & $0.58 \pm 0.15^{*}$ & 0.005 & 0.225 \\
\hline UP, ms/mmHg & $15.1 \pm 6$ & $18 \pm 11$ & $11.7 \pm 6.7^{*} \dagger$ & 0.738 & 0.008 \\
\hline DOWN, ms $/ \mathrm{mmHg}$ & $16.5 \pm 5.6$ & $18.3 \pm 8.8$ & $12.7 \pm 7.5^{*} \dagger$ & 0.738 & 0.004 \\
\hline GAIN, ms/mmHg & $16.1 \pm 5.5$ & $18.3 \pm 9.3$ & $12.3 \pm 7.2^{*} \dagger$ & 0.687 & 0.003 \\
\hline
\end{tabular}

Values expressed as means $\pm S D$ : standard deviation; BEI: baroreflex efficacy index; GAIN: total gain; DOWN: hypotensive responses associated with tachycardia responses; UP: hypertensive responses associated with bradycardic responses; significant difference $p<0.05$; ( $\left.{ }^{*}\right)$ vs rest Control, ( $\dagger$ ) vs rest eutrophic PCOS; P: eutrophic control group vs PCOS eutrophic group; Pll: PCOS eutrophic group vs. PCOS obese group.

Finally, in relation to BPV similarity were found between the studied groups, there are few information since there are no studies in the literature about the behaviour of BPV in PCOS women, the studies found are associated with cardiovascular diseases, unrelated to PCOS. ${ }^{29-31}$ Although, PCOS women have a greater predisposition to develop cardiovascular diseases, the present study population were healthy and did not use medication, suggesting that PCOS does not alter the BPV. In addition, the obese PCOS group also did not present differences in relation to eutrophic groups. The studies found on BPV and obesity are contradictory, some suggest an increase ${ }^{24,32}$ while others point out a reduction of BPV. ${ }^{33}$ However, both suggest that the baroreflex could justify these changes. Meanwhile, in our study, although the obese PCOS group presented a decrease in BRS, the BPV, apparently, was not affected. In this way, we need more studies to elucidate these findings.

\section{Study limitations}

The present study had some limitations, as insulin, glucose and inflammatory markers dosages absence, which could contribute to results discussion; another limitation was HRV and BPV measure only in supine position. It is possible that during an autonomic provocation test, as in tilt test, we could find different responses in autonomic modulation between the studied groups. However, it is important to note that the study limitations do not invalidate the main findings in supine position and its clinical implications. 


\section{Conclusion}

Although PCOS is an endocrine-metabolic disease that causes several body changes, it does not alter the autonomic cardiovascular control. However, the association with obesity resulted in a decrease in BRS values, and attenuated the HRV values. Suggesting that obesity may play a role in change hemodynamics parameters and cardiovascular autonomic control. However, further studies should be conducted to investigate the effects of metabolic and hormonal changes in these women and the association of these changes with cardiovascular autonomic control.

\section{Author contributions}

Conception and design of the research: Philbois SV, Souza HCD; acquisition of data: Philbois SV, Facioli TP, Felix ACS; analysis and interpretation of the data and critical revision of the manuscript for intellectual content: Philbois SV, Gastaldi AC, Souza HCD; statistical analysis: Philbois SV, Facioli TP; obtaining funding: Souza HCD; writing of the manuscript: Gastaldi AC, Souza HCD.

\section{References}

1. Yildirir A, Aybar F, Kabakci G, Yarali H, Oto A. Heart rate variability in young women with polycystic ovary syndrome. Ann Noninvasive Electrocardiol. 2006;11(4):306-12.

2. Tekin G, Tekin A, Kiliçarslan EB, Haydardedeoğlu B, Katircibaşi T, Koçum T, et al. Altered autonomic neural control of the cardiovascular system in patients with polycystic ovary syndrome. Int J Cardiol. 2008;130(1):49-55.

3. de Sá JC, Costa EC, da Silva E, Zuttin RS, da Silva EP, Lemos TM, et al. Analysis of heart rate variability in polycystic ovary syndrome. Gynecol Endocrinol. 2011;27(6):443-7.

4. Saranya K, Pal GK, Habeebullah S, Pal P. Assessment of cardiovascular autonomic function in patients with polycystic ovary syndrome. J Obstet Gynaecol Res. 2014;40(1):192-9.

5. La Rovere MT, Bigger JT, Marcus FI, Mortara A, Schwartz PJ. Baroreflex sensitivity and heart-rate variability in prediction of total cardiac mortality after myocardial infarction. ATRAMI (Autonomic Tone and Reflexes After Myocardial Infarction) Investigators. Lancet. 1998;351(9101):478-84.

6. Tank J, Jordan J, Diedrich A, Obst M, Plehm R, Luft FC, et al. Clonidine improves spontaneous baroreflex sensitivity in conscious mice through parasympathetic activation. Hypertension. 2004;43(5):1042-7.

7. Kouchaki Z, Butlin M, Qasem A, Avolio AP. Assessment of baroreflex sensitivity by continuous noninvasive monitoring of peripheral and central aortic pressure. Conf Proc IEEE Eng Med Biol Soc. 2014;2014:2940-3.

8. Kuppusamy S, Pal GK, Habeebullah S, Ananthanarayanan PH, Pal P. Association of sympathovagal imbalance with cardiovascular risks in patients with polycystic ovary syndrome. Endocr Res. 2015;40(1):37-43.

9. Sztajzel J, Golay A, Makoundou V, Lehmann TN, Barthassat V, Sievert K, et al. Impact of body fat mass extent on cardiac autonomic alterations in women. Eur J Clin Invest. 2009;39(8):649-56.

10. Thaisa HRDS, Gastaldi AC, Izabela CC, João EA, Suenimeire V, Hugo CDS. Effects of Physical Training on Cardiac Modulation in Normal Weight, Overweight, and Obese Individuals: A Comparative Study. J Nutr Food Sci. 2015:5(6):1-7.

\section{Potential Conflict of Interest}

No potential conflict of interest relevant to this article was reported.

\section{Sources of Funding}

This study was funded by CNPQ 457216/2014-0.

\section{Study Association}

This article is part of the thesis of master submitted by Stella Vieira Philbois, from Faculdade de Medicina de Ribeirão Preto.

\section{Ethics approval and consent to participate}

This study was approved by the Ethics Committee of the Hospital das Clínicas de Ribeirão Preto e da Faculdade de Medicina de Ribeirão Preto under the protocol number $11487 / 2014$. All the procedures in this study were in accordance with the 1975 Helsinki Declaration, updated in 2013. Informed consent was obtained from all participants included in the study.

11. Lambert EA, Teede $\mathrm{H}$, Sari $\mathrm{Cl}$, Jona $\mathrm{E}$, Shorakae $\mathrm{S}$, Woodington $\mathrm{K}$, et al Sympathetic activation and endothelial dysfunction in polycystic ovary syndrome are not explained by either obesity or insulin resistance. Clin Endocrinol (Oxf). 2015;83(6):812-9.

12. Rotterdam ESHRE/ASRM-Sponsored PCOS consensus workshop group. Revised 2003 consensus on diagnostic criteria and long-term health risks related to polycystic ovary syndrome (PCOS). Hum Reprod. 2004;19(1):41-7.

13. Griffin IJ, Cole TJ, Duncan KA, Hollman AS, Donaldson MD. Pelvic ultrasound measurements in normal girls. Acta Paediatr. 1995;84(5):536-43.

14. World Health Organization (WHO). Global status report on noncommunicable diseases 2010: Description of the global burden of NCDs, their risk factors and determinants. Geneva: World Health Organization; 2011.

15. Welch Be, Riendeau Rp, Crisp Ce, Isenstein Rs. Relationship of maximal oxygen consumption to various components of body composition. J Appl Physiol. 1958;12(3):395-8.

16. van de Borne P, Montano N, Zimmerman B, Pagani M, Somers VK. Relationship between repeated measures of hemodynamics, muscle sympathetic nerve activity, and their spectral oscillations. Circulation. 1997;96(12):4326-32.

17. Billman GE. Heart rate variability - a historical perspective. Front Physiol. 2011 Nov 29;2:86.

18. Heart rate variability: standards of measurement, physiological interpretation and clinical use. Task Force of the European Society of Cardiology and the North American Society of Pacing and Electrophysiology. Circulation. 1996;93(5):1043-65.

19. Montano N, Ruscone TG, Porta A, Lombardi F, Pagani M, Malliani A Power spectrum analysis of heart rate variability to assess the changes in sympathovagal balance during graded orthostatic tilt. Circulation. 1994;90(4):1826-31. 


\section{Original Article}

20. Di Rienzo M, Bertinieri G, Mancia G, Pedotti A. A new method for evaluating the baroreflex role by a joint pattern analysis of pulse interval and systolic blood pressure series. Med Biol Eng Comput. 1985;23:313-4.

21. Rowland TW. Effects of obesity on aerobic fitness in adolescent females. Am J Dis Child. 1991;145(7):764-8.

22. Ozcelik O, Aslan M, Ayar A, Kelestimur H. Effects of body mass index on maximal work production capacity and aerobic fitness during incremental exercise. Physiol Res. 2004;53(2):165-70.

23. Di Domenico K, Wiltgen D, Nickel FJ, Magalhães JA, Moraes RS, Spritzer PM. Cardiac autonomic modulation in polycystic ovary syndrome: does the phenotype matter? Fertil Steril. 2013;99(1):286-92.

24. Piccirillo G, Vetta F, Viola E, Santagada E, Ronzoni S, Cacciafesta M, et al Heart rate and blood pressure variability in obese normotensive subjects. Int J Obes Relat Metab Disord. 1998;22(8):741-50.

25. Piccirillo G, Vetta F, Fimognari FL, Ronzoni S, Lama J, Cacciafesta M, et al. Power spectral analysis of heart rate variability in obese subjects: evidence of decreased cardiac sympathetic responsiveness. Int J Obes Relat Metab Disord. 1996;20(9):825-9.

26. Indumathy J, Pal GK, Pal P, Ananthanarayanan PH, Parija SC, Balachander J, et al. Decreased baroreflex sensitivity is linked to sympathovagal imbalance, body fat mass and altered cardiometabolic profile in pre-obesity and obesity. Metabolism. 2015;64(12):1704-14.
27. Skrapari I, Tentolouris N, Katsilambros N. Baroreflex function: determinants in healthy subjects and disturbances in diabetes, obesity and metabolic syndrome. Curr Diabetes Rev. 2006; 2(3):329-38.

28. De Melo VU, Saldanha RR, Dos Santos CR, De Campos Cruz J, Lira VA Santana-Filho VJ, et al. Ovarian Hormone Deprivation Reduces Oxytocin Expression in Paraventricular Nucleus Preautonomic Neurons and Correlates with Baroreflex Impairment in Rats. Front Physiol. 2016 Oct 13;7:461.

29. Mancia G, Ferrari A, Gregorini L, Parati G, Pomidossi G, Bertinieri G, et al. Blood pressure and heart rate variabilities in normotensive and hypertensive human beings. Circ Res. 1983;53(1):96-104.

30. Heusser K, Tank J, Engeli S, Diedrich A, Menne J, Eckert S, et al. Carotid baroreceptor stimulation, sympathetic activity, baroreflex function, and blood pressure in hypertensive patients. Hypertension. 2010;55(3):619-26.

31. Rothwell PM, Howard SC, Dolan E, O'Brien E, Dobson JE, Dahlöf B, et al. Prognostic significance of visit-to-visit variability, maximum systolic blood pressure, and episodic hypertension. Lancet. 2010;375(9718):895-905.

32. Lucini D, de Giacomi G, Tosi F, Malacarne M, Respizzi S, Pagani M. Altered cardiovascular autonomic regulation in overweight children engaged in regular physical activity. Heart. 2013;99(6):376-81.

33. Quilliot D, Fluckiger L, Zannad F, Drouin P, Ziegler O. Impaired autonomic control of heart rate and blood pressure in obesity: role of age and of insulinresistance. Clin Auton Res. 2001;11(2):79-86. 